\title{
Somogy megye ormányosalkatú bogarainak katalógusa (Coleoptera: Curculionoidea)
}

\author{
Podlussány AtTtila
}

Podlussány A: Catalogue of the weevil fauna of Somogy county (Coleoptera: Curculionoidea)

Abstract: The author has gathered the data of the Curculionoidea species collected in the Somogy county from 1886 till nowadays. Since no investigation was ever made on the whole county, the description of 757 species is a significant result, which shows, the richness of this county's flora, being the species herbivorous. The author mentions a new species for the Hungarian fauna: Ceutorhynchus fulvitarsis Gougelet \& H. Brisout de Barneville, 1860, found in Somogy county till now.

\section{Bevezetés}

Egy katalógus összeállításakor még a mai számítógépes világunkban is komoly irodalmi áttekintést kell végezni. Krécsy Béla már megemlít néhány ormányosalkatú bogarat Somogy megyéból (KRÉCSY 1886). Kuthy Dezsôtól a Fauna Regni Hungariae címú munkában is találtam somogyi adatokat (KUTHY 1896). Sajnos Kuthy a gyakori fajoknál nem sorolta fel a lelóhelyeket, így csak a ritkább fajok Somogy megyei adatait tudtam kigyújteni. Endrődi Sebó Kárpát-medencei lelóhelyadatai között már jelentôs mennyiségú somogyi adatot közölt (ENDRÓdI 1957, 1958b, 1959a, 1960b, 1961c, 1963a, 1969, 1970). Magam részt vettem a Duna-Dráva N. P. Dráva-mente faunakutatásában, ahonnan jelentôs számú (327) faj került eló, mely lelóhelyadatok nagyobb része Somogy megyéból származik (PoDlussínY 1998). Feldolgoztam továbbá a Janus Pannonius Múzeum Curculionoidea gyújteményét, melyben a Zselici TK-ból és a Barcsi ősborókásból származó néhány adatot találtam. Felhasználtam még a közelmúlt szórványgyújitéseinek adatait is. A még nem publikált gyứjtési adatokat „elsô közlés“ szavakkal jelöltem. A listában található egy, Magyarország faunájára új faj is, a Ceutorhynchus fulvitarsis Gougelet \& $H$. Brisout de Barneville, 1860. A faj gyúijtési adatai a következók: Vízvár, Dráva-part, 1995. IX. 6-7. leg. Podlussány Attila; Vízvár, Zsitvai-rét, 1998. VI. 12. leg. Kondorossy Előd. Kérdőjellel jelöltem a taxonómia szempontjából problematikus, illetve a feltételezett kétes eloofordulású fajneveket. A listában a jelenleg Európa-szerte érvényes neveket használtam (PoDlussánY 1996). Összegezve a listában szereplô fajok száma 757.

Meglepó, és aki nap mint nap faunisztikával foglalkozik, annak gyanús is ez az igen magas fajszám, mivel jól tudjuk, hogy egész Somogy megyét átfogó faunakutató program az elmúlt 120 év alatt nem volt. A listában felsorolt fajok gyüjtési adatainak 52\%-a 50 évnél idôsebb. Nem feltételezem azt, hogy elődeink tévesen közöltek lelóhelyadatokat, illetve tévesen determináltak, azonban ezek az adatok szerintem megerősítésre szorulnak. Jó lenne megbizonyosodni róla, hogy élnek-e ezek a fajok Somogy megyében ma is, vagy eltúntek. Egy alapos, törvényi korlátok nélküli, szakemberek által végzett, gyújtő és feldol- 
gozó programot kellene végrehajtani. Sok pénz, 12 szakember és 5 év kell hozzá. Sajnos a jelenlegi pénzhiány, a természetvédelmi korlátok, valamint a drasztikus gyújtő- és kutatóhiány miatt, Somogy megye faunakutatása a jövóben is csak ábránd marad.

\section{Somogy megye területéról kimutatott fajok listája}

\section{Curculionidae \\ Acalles camelus (Fabricius, 1792) - Endródi 1961 \\ Acalles commutatus Dieckmann, 1982 . \\ Podlussány 1998 \\ Acalles echinatus (Germar, 1824). \\ Podlussány 1998}

?Acalles lemur (Germar, 1824) - Endrödi 1961

Acalles parvulus Boheman, 1837 - Endródi 1961

Acalles roboris Curtis, 1835 - elsö közlés

Acallocrates denticollis (Germar, 1824) -

Endródi 1961

Acalyptus carpini (Fabricius, 1792).

Podlussány 1998

Acalyptus sericeus Gyllenhal, 1836

Podlussány 1998

Adexius scrobipennis Gyllenhal, 1834 .

Endrốdi 1961

Adosomus roridus (Pallas, 1781) - Endródi 1959

Alophus kaufmanni Stierlin, 1884 - Endrődi 1961

Alophus triguttatus (Fabricius, 1775) -

Podlussány 1998

Amalorrhynchus melanarius (Stephens,

1831) - Endrödi 1969

Amalus scortillum (Herbst, 1795) - elsó közlés

Anthonomus bituberculatus Tomson, 1868 .

Endrödi 1970

Anthonomus pedicularius (Linné, 1758) -

Krécsy 1886, Endródi 1970, Podlussány 1998

Anthonomus phyllocola (Herbst, 1795) .

Endrödi 1970

Anthonomus piri Kollar, 1837 - Endródi 1970

Anthonomus pomorum (Linné, 1758) -

Endrödi 1970

Anthonomus rubi (Herbst, 1795) - Endrödi

1970, Podlussány 1998

Anthonomus rufus Gyllenhal, 1836 .

Podlussány 1998

Anthonomus ulmi (De Geer, 1775) - Endrődi 1970, Podlussány 1998

Aphytobius sphaerion (Boheman, 1845) -
Endrődi 1969

Auleutes epilobii (Paykull, 1800)

Podlussány 1998

Axenomimetes reflexus (Boheman, 1838).

Kuthy 1896, Endrődi 1961

Bagous angustus Silfverberg, 1977 - Endródi 1970

Bagous argillaceus Gyllenhal, 1836 - Endrödi 1970, Podlussány 1998

Bagous bagdatensis Pic, 1904 - Podlussány 1998

Bagous collignensis (Herbst, 1797) - Endródi 1970, Podlussány 1998

Bagous friwaldszkyi Tournier, 1874 - elsö közlés

Bagous glabrirostris (Herbst, 1795) -

Endrôdi 1970

Bagous limosus (Gyllenhal, 1827) - Endródi 1970

Bagous longitarsis Thomson, 1868 - Endrôdi 1970 1970

Bagous lutosus (Gyllenhal, 18I3) - Endrödi

Bagous lutulentus (Gyllenhal, 1813). Endrödi 1970, Podlussány 1998

Bagous lutulosus (Gyllenhal, 1827)

Endrődi 1970 1970

Bagous nodulosus Gyllenhal, 1836 - Endrődi

Bagous petro (Herbst, 1795) - Endrödi 1970

Bagous puncticollis Boheman, 1845. Podlussány 1998

Bagous robustus H. Brisout de Barneville, 1863 - Endrōdi 1970

Bagous subcarinatus Gyllenhal, 1836 . Endrödi 1970, Podlussány 1998

Bagous tempestivus (Herbst, 1795) - Endrődi 1970, Podlussány 1998 1970

Bagous validus Rosenhauer, 1847 - Endródi

Baris analis (Olivier, 1790) - Endrődi 1969

Baris artemisiae (Herbst, 1795) - Endródi 1969, Podlussány 1998

Baris angusta Brullé, 1832 - Endródi 1969 
Baris atramentaria (Boheman, 1836) Endrödi 1969

Baris chlorizans Germax, 1824 - Endrödi 1969

Baris coerulescens (Scopoli, 1763) - Endrődi 1969, Podlussány 1998

Baris gudenusi Schultze, 1901 - Endródi 1969 1969

Baris laticollis (Marsham, 1802) - Endrődi

Baris lepidii Germar, 1824 - Endrődi 1969 1969

Baris picicomis (Marsham, 1802) - Endrödi

Baris scolopacea Germar, 1824 - Endródi 1969

Baris timida (Rossi, 1792) - Endródi 1969

Baris villae Comolli, 1837 - Endrődi 1969, Podlussány 1998

Barynotus obscurus (Fabricius, 1775) Endrốdi 1959

Barypeithes araneiformis (Schrank, 1781) - elsö közlés

Barypeithes cherrolati (Boheman, 1843) -

Endrödi 1959, Podlussány 1998

Barypeithes mollicomus (Ahrens, 1812) -

Kuthy 1896, Endrődi 1959

Barypeithes styriacus Seidlitz, 1868 -

Endrődi 1959

Bothynoderes punctiventris (Germar, 1824) Endrôdi 1959, Podlussány 1998

Brachonyx pineti (Paykull, 1792) - Endrődi 1970, Podlussány 1998

Brachyderes incanus (Linné, 1758) -

Podlussány 1998

Brachycerus foreicollis Gyllenhal, 1833 .

Endrödi 1960

Brachysomus echinatus (Bonsdorff, 1785) -

Endrốdi 1959

Brachysomus hirtus (Boheman, 1845) -

Endrődi 1959

Brachysomus setiger (Gyllenhal, 1840) .

Endrốdi 1959, Podlussány 1998 közlés

Brachysomus villosulus (Germar, 1824) - elsö

Brachytemnus porcatus (Germar, 1824).

Endrődi 1961, Podlussány 1998 1970

Bradybatus creutzeri Germar, 1824 - Endrödi

Bradybatus fallax Gerstäcker, 1860 . Endrődi 1970

Bradybatus kellneri Bach, 1854 - Endrődi 1970

Calosirus terminatus (Herbst, 1795).

Endródi 1969

Camptorhinus statua (Rossi, 1790) - Endrôdi 1961

Ceutorhynchus aeneicollis Germar, 1824 Endrốdi 1969

Ceutorhynchus alliariae $\mathrm{H}$. Brisout de
Barneville, 1860 - Endrődi 1969

Ceutorhynchus assimilis (Paykull, 1792)

(=pleurostigma) - Endrödi 1969

Ceutorhynchus atomus Boheman, 1845 .

Endrődi 1969, Podlussány 1998

Ceutorhynchus canaliculatus Ch. Brisout de

Barneville, 1869 - Endrốdi 1969

Ceutorhynchus carinatus Gyllenhal, 1837 .

Endrödi 1969

Ceutorhynchus chalybaeus Germar, 1824 .

Endrödi 1969

Ceutorhynchus coarctatus Gyllenhal, 1837 .

Endrődi 1969

Ceutorhynchus cochleariae (Gyllenhal, 1813) .

Endrödi 1969, Podlussány 1998

Ceutorhynchus constrictus (Marsham, 1802) -

Endrốdi 1969

Ceutorhynchus contractus (Marsham, 1802) -

Endródi 1969, Podlussány 1998

Ceutorhynchus dubius Ch. Brisout de

Barbeville, 1883 - Endrödi 1969

Ceutorhynchus erysimi (Fabricius, 1787) -

Endrödi 1969, Podlussány 1998

Ceutorhynchus floralis (Paykull, 1792) -

Endrődi 1969, Podlussány 1998

Ceutorhynchus fulvitarsis Gougelet \& $\mathrm{H}$.

Brisout de Barneville, 1860 - faunánkban új, elsö közlés

Ceutorhynchus griseus Ch. Brisout de Barneville, 1869 - Endrődi 1969

Ceutorhynchus hampei (Ch. Brisout de

Barneville, 1869) - Endródi 1969

Ceutorhynchus hirtulus Germar, 1824 .

Endrốdi 1969

Ceutorhynchus ignitus Germar, 1824 .

Endrödi 1969

Ceutorhynchus inaffectatus Gyllenhal, 1937 -

Endrődi 1969

Ceutorhynchus interjectus Schultze, 1903 -

Endrődi 1969

Ceutorhynchus liliputanus Schultze, 1898 -

Endrődi 1969

Ceutorhynchus nanus Gyllenhal, 1837 -

Endrödi 1969

Ceutorhynchus napi Gyllenhal, 1837 .

Endrődi 1969

Ceutorhynchus obstrictus (Marsham, 1802) -

Kuthy 1896, Endrődi 1969, Podlussány 1998

Ceutorhynchus pallidactylus (Marsham, 1802) - Endródi 1969, Podlussány 1998

Ceutorhynchus pandellei Ch. Brisout de

Barneville, 1869 - Endrôdi 1969

Ceutorhynchus pervicax Weise, 1883 - elsö közlés

Ceutorhynchus picitarsis Gyllenhal, 1837 .

Endrödi 1969 
Ceutorhynchus posthumus Germar, 1824 .

Endrödi 1969, Podlussány 1998

Ceutorhynchus pulvinatus (Gyllenhal, 1837) -

Kuthy 1896, Endrödi 1969

Ceutorhynchus puncticollis Boheman, 1845 -

Endródi 1969, Podlussány 1998

Ceutorhynchus rapae Gyllenhal, 1837 -

Kuthy 1896, Endrôdi 1969

Ceutorhynchus roberti Gyllenhal, 1837 .

Endrödi 1969

Ceutorhynchus scapularis Gyllenhal, 1837 -

Endrödi 1969

?Ceutorhynchus schoenherri Ch. Brisout de

Barneville, 1869 - Endródi 1969

Ceutorhynchus sisymbrii Dieckmann, 1966 elsô közlés

Ceutorhynchus sophiae (Steven, 1829).

Kuthy 1896, Endrôdi 1969

Ceutorhynchus sulcatus Ch. Brisout de Barneville, 1869 - Endrödi 1969

Ceutorhynchus sulcicollis (Paykull, 1800) -

Kuthy 1896, Endrôdi 1969

Ceutorhynchus syrites Germar, 1824 .

Endrốdi 1969

Ceutorhynchus turbatus Schulize, 1903.

Endrödi 1969

Ceutorhynchus unguicularis Thomson, 187I .

Endrödi 1969

Ceutorhynchus viridanus Gyllenhal, 1837 .

Endrödi 1969

Chlorophanus graminicola Schönherr, 1832 .

Kuthy I896, Endrődi 1959, Podlussány 1998

Chlorophanus pollinosus (Fabricius, 1792) -

Kuthy 1896, Endrödi 1959

Chromoderus affinis (Schrank, 1781) -

Endrốdi 1959

Chromoderus declivis (Olivier, 1807) .

Endrödi 1959

Cionus alauda (Herbst, 1784) - Endródi 1970

Cionus clairvillei Boheman, 1838 - Endrődi 1970

Cionus hortulanus (Fourcroy, 1785) -

Endrôdi 1970, Podlussány 1998

Cionus leonhardi Wingelmüller, 1914. Endrődi 1970

Cionus longicollis montanus Wingelmüller,

1914 - Endródi 1970

Cionus nigritarsis Reitter, 1904 - Endródi 1970

Cionus olens (Fabricius, 1798) - Endrödi 1970

Cionus olivieri Rosenschöld, 1838 - Endrődi

1970, Podlussány 1998 1970

Cionus scrophulariae (Linné, 1758) - Endrődi

Cionus thapsus (Fabricius, 1792) - Endrôdi 1970, Podlussány 1998
Cionus tuberculosus (Scopoli, 1792) Endrődi 1970

Cleonis pigra (Scopoli, 1763) - Endródi 1959, Podlussány 1998

Cleopus solani (Fabricius, 1792) - Endrôdi

1970, Podlussány 1998

Coeliastes lamii (Fabricius, 1792) - Endródi 1969, Podlussány 1998

Coeliodes erythroleucos (Gmelin, 1790) Endrōdi 1969 1969

Coleiodes dryados (Gmelin, 1790) - Endrődi

Coeliodes proximus Schultze, 1895 - Endrödi 1969

Coeliodes ruber (Marsham, 1802) - Endrődi 1969

Coeliodes rubicundus (Herbst, 1795). Endrődi 1969

Coeliodes trifasciatus Bach, 1854 - Endrödi 1969

Coniocleonus nigrosuturatus (Goeze, 1777) Endrödi 1959

Coniocleonus turbatus (Fahraeus, 1842) - elsó közlés

Coryssomerus capucinus (Beck, 1817 Endrödi 1969

Cossonus cylindricus C. R. Sahlberg, 1835 Endrő́di 1961 1961

Cossonus linearis (Fabricius, 1775) - Endrődi

Cryptorhynchus lapathi (Linné, 1758) -

Endrớdi 1961, Podlussány 1998

Ctenochirus leucogrammus (Germar, 1824) Endrốdi 1960

Curculio betulae (Stephens, 1831). Podlussány 1998

Curculio crux Fabricius, 1776 - Endrödi 1970, Podlussány 1998 1970

Curculio elephas (Gyllenhal, 1836) - Endrödi

Curculio glandium Marsham, 1802 - Endrődi 1970, Podlussány 1998

Curculio nucum Linné, 1758 - Podlussány 1998

Curculio propinquus (Desbrochers des Loges, 1868) - Endrődi 1970

Curculio pyrrhoceras Marsham, 1802 -

Endrődi 1970, Podlussány 1998

Curculio saliciporus (Paykull, 1792) -

Endrődi 1970, Podlussány 1998

Curculio venosus (Gravenhorst, 1807) -

Endrődi 1970, Podlussány 1998

Curculio pillosus Fabricius, 1781 - Endrődi 1970 
Cycloderes pilosulus (Herbst, 1795) -

Endrődi 1959, Podlussány 1998

Cyphocleonus achates (Fahraeus, 1842) -

Endrődi 1959

Cyphocleonus dealbatus (Gmelin, 1790) -

Endrődi 1959

Datonychus angulosus (Boheman, 1845) -

Endrốdi 1969, Podlussány 1998

Datonychus arquatus (Herbst, 1795) -

Endrödi 1969

Datonychus frivaldszkyi (Schultze, 1896) -

Endrödi 1969

Datonychus melanostictus (Marsham, 1802) -

Endrődi 1969

Datonychus paszlanszkyi (Kuthy, 1890) -

Endrődi 1969

Donus oxalis (Herbst, 1795) - Endrödi

1961, Podlussány 1998

Donus maculatus (W. Redtenbacher, 1842) -

Endrődi I961

1970

Dorytomus affinis (Paykull, 1800) - Endrődi

Dorytomus dejeani Faust, 1882 - Endródi 1970 1970

Dorytomus dorsalis (Linné, 1795) - Endrődi

Dorytomus filirostris (Gyllenhal, 1836) -

Endrôdi 1970, Podlussány 1998

Dorytomus hirtipennis Bedel, 1884 - Endrődi

1970, Podlussány 1998

Dorytomus ictor (Herbst, 1795) - Endródi 1970

Dorytomus longimanus (Forster, 1771) -

Endrődi 1970, Podlussány 1998

Dorytomus majalis (Paykull, 1800) -

Podlussány 1998

Dorytomus melanophthalmus (Paykull, 1792) -

Kuthy 1896, Endrödi 1970, Podlussány 1998

Dorytomus minutus (Gyllenhal, 1836) -

Endródi 1970, Podlussány 1998

Dorytomus nebulosus (Gyllenhal, 1836) .

Endrốdi 1970

Dorytomus occallescens (Gyllenhal, 1836).

Podlussány 1998

Dorytomus rufatus (Bedel, 1888) - Endrődi

1970, Podlussány 1998

Dorytomus salicis Walton, 1851 - Endródi 1970

Dorytomus schoenherri Faust, 1883 - Endrődi

1970, Podlussány 1998

Dorytomus suratus (Gyllenhal, 1836) -

Endrődi 1970

Dorytomus taeniatus (Fabricius, 1781) -

Endrődi 1970, Podlussány 1998

Dorytomus tremulae (Fabricius, 1787) -

Endrōdi 1970, Podlussány 1998

Dorytomus villosulus (Gyllenhal, 1836) -

Podlussány 1998
Dryophthorus corticalis (Paykull, 1792) Kuthy 1896, Endrödi 1961

Echinocnemus globicollis Fairmaire, 1863 -

Endrốdi 1970 1970

Ellescus bipunctatus (Linné, 1758) - Endrödi

Ellescus infirmus (Herbst, 1795) - Endrődi 1970, Podlussány 1998

Ellescus scanicus (Paykull, 1792) - Endródi 1970

Ethelcus denticulatus (Schrank, 1781) Endrődi 1969

Eubrychius velutus (Beck, 1817) - Endrödi 1969

Eucoeliodes mirabilis (A. Villa \& G. B. Villa, 1835) - elsö közlés

Eusomus ovulum Germar, 1824 - Endrődi 1959, Podlussány 1998

Furcipus rectirostris (Linné, 1758) - Endródi 1970, Podlussány 1998

Gasterocercus depressirostris (Fabricius, 1792) Endrổi 1961

Glocianus distinctus (Ch. Brisout, 1870) Endrődi 1969

Glocianus fennicus (Faust, 1894) - Endrödi 1969

Glocianus moelleri (Thomson, 1868) - Kuthy 1896, Endródi 1969

Glocianus pilosellus (Gyllenhal, 1837) -

Endrớdi 1969

Glocianus punctiger (Gyllenhal, 1837) Endrődi 1969

Grypus equiseti (Fabricius, 1775) -

Podlussány 1998

Gymnetron antirrhini (Paykull, 1800) -

Endrốdi 1970, Podlussány 1998

Gymnetron asellus (Gravenhorst, 1807) -

Endródi 1970, Podlussány 1998

Gymnetron beccabungae (Linné, 1761) .

Endrődi 1970

Gymnetron bipustulatum (Rossi, 1794) -

Endródi 1970, Podlussány 1998

Gymnetron collinum (Gyllenhal, 1813) .

Endrớdi 1970 1970

Gymnetron hispidum Brullé, 1832 - Endrődi

Gymnetron ictericum Gyllenhal, 1838 -

Endródi 1970

Gymnetron labile (Herbst, 1795) - Endrôdi

1970, Podlussány 1998

1970

Gymnetron linariae (Panzer, 1796) - Endrődi

Gymnetron littoreum $\mathrm{H}$. Brisout de

Barneville, 1862 - Podlussány 1998

Gymnetron melanarium (Germar, 1821) -

Endrődi 1970, Podlussány 1998 

1970

Gymnetron melas Boheman, 1838 - Endrődi

Gymnetron netum (Germar, I82I) - Kuthy 1896, Endródi 1970

Gymnetron pascuorum (Gyllenhal, 1813) . Endrốdi 1970), Podlussány 1998

Gymnetron pirrazolii (Stierlin, 1867). Endrődi 1970, Podlussány 1998

Gymnetron rostellum (Herbsi, 1795) - Kuthy 1896, Endrődi 1970

Gymnetron stimulosum (Germar, 1821) -

Endródi 1970

Gymnetron tetrum (Fabricius, 1792) - Kuthy

1896, Endródi 1970, Podlussány 1998

Gymnetron veronicae (Germar, I821) -

Endródi 1970

Gymnetron villosulum Gyllenhal, 1838 -

Endródi 1970

Hadroplontus trimaculatus (Fabricius, 1775) -

Endrödi 1969

Hexarthrum exiguum (Boheman, 1838) -

Endrődi 1961

Hexarthrum truncorum (Germar, I 824) -

Endrödi 1961

Homorosoma validirostre (Gyllenhal, 1837) -

Endrốdi 1969

Hydronomus alismatis (Marsham, 1802) -

Endrốdi 1970

Hylobitelus abietis (Linné, 1758) - Endrődi

1961, Podlussány 1998

Hylobitelus transyersovittatus (Goeze, 1777) -

Endrốdi 1961 1961

Hypera adspersa (Fabricius, 1792) - Endrődi

Hypera arator (Linné, 1758) - Endrödi 1961 1961

Hypera arundinis (Paykull, 1792) - Endrődi

Hypera contaminata (Herbst, 1795) .

Endrôdi 1961, Podlussány 1998

Hypera cumana (Petri, 1901) - Endrődi 1961

Hypera dauci (Olivier, 1807) - Endrödi 1961

Hypera denominanda (Capiomont, 1868) -

Endrö́di 1961

Hypera diversipunctata (Schrank, 1798) -

Endrôdi 1961, Podlussány 1998

Hypera fuscocinerea (Marsham, 1802).

Endrödi 1961

Hypera meles (Fabricius, 1792) - Endrődi

1961, Podlussány 1998

Hypera nigrirostris (Fabricius, 1775) -

Endrôdi 1961, Podlussány 1998

Hypera plantaginis (De Geer, 1775) .

Endrö́di 1961 1961

Hypera postica (Gyllenhal, 1813) - Endródi
Hypera rumicis (Linné, 1758) - Endrödi 1961, Podlussány 1998

Hypera striata (Boheman, 1834) - Endrôdi

1961, Podlussány 1998

Hypera suspiciosa (Herbst, 1795) - Endródi 1961 1961

Hypera venusta (Fabricius, 1781) - Endródi

Hypera riciae (Gyllenhal, 1813) - Endrödi 1961

Hypera zoilus (Scopoli, 1763) - Endrödi 1961

Icaris sparganii (Gyllenhal, 1836) - Endródi 1970

Isochnus populicola Silfverberg, 1977 -

Endrődi 1970, Podlussány 1998

Lachnaeus crinitus (Boheman, 1836) -

Endrớdi 1959

Larinus brevis (Herbst, 1795) - Endrödi 1959

Larinus jaceae (Fabricius, 1775) - Endrődi 1959

Larinus minutus Gyllenhal, 1836 - Endródi 1959

Larinus obtusus Gyllenhal, 1836 - Endrödi 1959, Podlussány 1998

Larinus planus (Fabricius, 1792) - Endródi 1959, Podlussány 1998

Larinus sturnus (Schaller, 1783) - Endrődi

1959, Podlussány 1998

Larinus turbinatus Gyllenhal, I836 -

Endródi 1959, Podlussány 1998

Leiosoma deflexum (Panzer, 1794) - Endrődi 1961

Lepyrus capucinus (Schaller, 1783) - Endrődi 1961, Podlussány 1998

Lepyrus palustris (Scopoli, 1763) - Endrōdi 1961, Podlussány 1998

Leucosomus pedestris (Poda, 1761) - Endródi 1959

Lignyodes enucleator (Panzer, 1798) Endrớdi 1970

Lignyodes suturatus Fairmaire, 1859 - elsó közlés

Lignyodes uniformis Desbrochers des Loges, 1894 - Endrődi 1970

Limobius borealis (Paykull, 1792) - Endrôdi 1961

Limnobaris dolorosa (Goeze, 1777) - Endrődi 1969

Limnobaris t-album (Linné, 1758) - Endrődi 1969, Podlussány 1998

?Liophloeus liptoviensis J. Weise, 1894 Endródi 1959

Liophloeus tessulatus (O. F. Müller, 1776) Endrődi 1959, Podlussány 1998 1961

Liparus coronatus (Goeze, 1777) - Endrődi

Liparus dirus (Herbst, 1795) - Endrödi 1961

Lixus angustatus (Fabricius, 1775) - Endrödi 1959 
Lizus angustus (Herbst, I795) - Endrődi 1959

?Lixus ascanii (Linné, 1767) - Endrödi 1959

Lixus bardanae (Fabricius, 1787) - Endrôdi

1959, Podlussány 1998

Lixus brevipes $\mathrm{Ch}$. Brisout de Barneville, 1866 - Endrődi 1959

Lixzıs cardui Olivier, 1808 - Endrödi 1959

Lixus elegantulus Boheman, 1843 - Endrődi 1959

Lixus fasciculatus Boheman, 1836 . Podlussány 1998

Lixus filiformis (Fabricius, 1781) - Endrödi 1959, Podlussány 1998

Lixus iridis Olivier, 1807 - Endródi 1959,

Podlussány 1998

Lixus myagri Olivier, 1807 - Endrödi 1959

Lixus paraplecticus (Linné, 1758) - Endrődi

1959, Podlussány 1998

Lixus punctirostris Boheman, 1843 - Endrödi 1959

Lixus punctiventris Boheman, 1836 -

Endródi 1959

Lixus subtilis Boheman, 1836 - Endrődi 1959, Podlussány 1998

Lixus vilis (Rossi, 1790) - Endrődi 1959

Magdalis armigera (Fourcroy, 1785) -

Endrődi 1961

Magdalis barbicornis (Latreille, 1804) -

Endrődi 1961

Magdalis cerasi (Linné, 1758) - Endrödi

1961, Podlussány 1998

Magdalis exarata (H. Brisout de Barneville,

1862) - Endrő́di 1961

Magdalis flavicomis (Gyllenhal, 1836) -

Endrődi 1961

Magdalis frontalis (Gyllenhal, 1827) -

Endrődi 1961

Magdalis memnonia (Gyllenhal, 1837) -

Endrơdi 1961, Podlussány 1998

Magdalis nitidipennis (Boheman, 1843) -

Endrődi 1961

Magdalis phlegmatica (Herbst, 1797) -

Podlussány 1998

Magdalis rufa Germar, 1824 - Endrôdi

1961, Podlussány 1998 1961

Magdalis ruficornis (Linné, 1758) - Endrödi 1961

Magdalis piolaceus (Linné, 1758) - Endrődi

Marmaropus besseri Gyllenhal, 1837 -

Endrödi 1969 1959

Mecaspis alternans (Herbst, 1795) - Endrődi

Mecinus collaris Germar, 1821 - Endródi 1970
Mecinus janthinus (Germar, 1817) - Endrődi 1970

Mecinus pyraster (Herbst, 1795) - Kuthy 1896, Endrö́di 1970

Mesagroicus obscurus Boheman, 1840 -

Endrődi 1959, Podlussány 1998

Miarus ajugae (Herbst, 1795) - Podlussány 1998

?Miarus campanulae (Linné, 1767) -

Endrődi 1970

Miarus graminis (Gyllenhal, 1813) -

Endrớdi 1970

Miarus micros (Germar, 1821) - Endródi 1970

Microplontus campestris (Gyllenhal, 1837) -

Endrődi 1969

Microplontus edentulus (Schultze, 1897) -

Endrődi 1969

Microplontus figuratus (Gyllenhal, 1837) - elsố közlés

Microplontus rugulosus (Herbst, 1795) -

Endrődi 1969

Minyops carinatus (Linné, 1767) - Endrödi 1961

Mogulones abbreviatulus (Fabricius, 1792) -

Endrö́di 1969, Podlussány 1998

Mogulones albosignatus (Gyllenhal, 1837) -

Endrődi 1969

Mogulones amplipennis (Schultze, 1896) -

Endrödi 1969

Mogulones asperifoliarum (Gyllenhal, 1813) -

Endrôdi 1969 1969

Mogulones aubei (Boheman, 1845) - Endrödi

Mogulones austriacus (Ch. Brisout de Barneville, 1869) - Kuthy 1896, Endrö́di 1969

Mogulones borraginis (Fabricius, 1792) Endrődi 1969

Mogulones cruciger (Herbst, 1784) - Endródi 1969

Mogulones dimidiatus (I. Frivaldszky, 1865) -

Endrődi 1969

Mogulones euphorbiae (Ch. Brisout de

Barneville, 1866) - Endródi 1969

Mogulones geographicus (Goeze, 1777) -

Endrődi 1969

Mogulones hungaricus (Ch. Brisout de Barneville, 1869) - Endrôdi 1969

Mogulones javeti (Ch. Brisout de Barneville, 1869) - Endrödi 1969

Mogulones larvatus (Schultze, 1897) -

Endrödi 1969

Mogulones ornatus (Gyllenhal, 1837) -

Endrődi 1969

Mogulones pallidicornis $(\mathrm{H}$. Brisout de Barneville, 1860) - Endrödi 1969

Mogulones raphani (Fabricius, 1792) (symphyti) - Endrốdi 1969, Podlussány 1998 
Mogulones trisignatus (Gyllenhal, 1837) -

Endródi 1969

Mogulonoides radula (Germar, 1824) -

Endródi 1969, Podlussány 1998

Mononychus punctumalbum (Herbst, 1784) -

Endródi 1969, Podlussány 1998

Myorhinus albolineatus (Fabricius, 1792) -

Endrödi 1961

Nedyus quadrimaculatus (Linné, 1758) -

Endrôdi 1969, Podlussány 1998

Neoglocianus albovittatus (Germar, 1824) -

Endródi 1969

Neoglocianus maculaalba (Herbst, 1795) -

Kuthy 1896, Endródi 1969

Neophytobius quadrinodosus (Gyllenhal,

I813) - Endrődi 1969

Neoplinthus tigratus (Rossi, 1792) - elsö közlés

Notaris acridulus (Linné, 1758) - Endrődi

1970, Podlussány 1998

Notaris bimaculatus (Fabricius, 1787) -

Endrôdi 1970

Notaris granulipennis Tournier, 1874 -

Podlussány 1998

Notaris scirpi (Fabricius, 1792) - Endródi 1970

Omias globulus (Boheman, 1843) - Endrődi

1960

Omias rotundatus (Fabricius, 1792) -

Endrödi 1960

Omias seminulum (Fabricius, 1792) -

Endrôdi 1960

Oprohinus consputus (Germar, 1824) -

Endrốdi 1969

Oprohinus suturalis (Fabricius, 1777) -

Endrödi 1969

Orobitis cyaneus (Linné, 1758) - Endrődi

1969, Podlussány 1998

Otiorhynchus austriacus (Fabricius, 180I) -

Podlussány 1998

?Otiorhynchus cardiniger Host, 1789 -

Endródi 1960

Otiorhynchus conspersus (Herbst, 1795) -

Endrödi 1960

Otiorhynchus duinensis Germar, 1824 -

Endrődi 1960, Podlussány 1998

Otiorhynchus fullo (Schrank, I781) -

Endródi 1960

Otiorhynchus hungaricus Germar, 1824 -

Endrödi 1960

Otiorhynchus inflatus Gyllenhal, 1834 -

Endrödi 1960, Podlussány 1998

Otiorhynchus laevigatus (Fabricius, 1775) -

Endródi 1960) Otiorhynchus lepidopterus (Fabricius, 1794) -

Endrödi 1960

Otiorhynchus ligustici (Linné, 1758) -
Endrődi 1960, Podlussány 1998

Otiorhynchus mandibularis W. Redtenbacher, 1842 - Endródi 1960

Otiorhynchus mastix (Olivier, 1807) -

Endrődi 1960, Podlussány 1998

Otiorhynchus multipunctatus (Fabricius,

1792) - Endrôdi 1960

Otiorhynchus orbicularis (Herbst, 1795) -

Endrödi 1960

Otiorhynchus ovatus (Linné, 1758) - Endrōdi

1960, Podlussány 1998

Otiorhynchus pinastri (Herbst, 1795).

Podlussány 1998

Otiorhynchus pulverulentus Germar, 1824 -

Endrödi 1960

Otiorhynchus raucus (Fabricius, 1776) -

Endródi 1960, Podlussány 1998

Otiorhynchus reichei Sticrlin, 1861.

Podlussány 1998

Otiorhynchus repletus Boheman, 1843 -

Endrödi 1960

Otiorhynchus rugosostriatus (Goeze, 1777) -

Endrődi 1960, Podlussány 1998

?Otiorhynchus signatipennis Gyllenhal, 1834 -

Krécsy 1886

Otiorhynchus pelutinus Germar, 1824 -

Endródi, I 960

Pachycerus cordiger (Germar, 1819) - Endródi

1959

Pachyrhinus mustela (Herbst, 1797) -

Endrödi 1959

Pachytychius sparsutus (Olivier, 1807) -

Endrődi 1970

Parafoucartia squamulata (Herbst, 1795) -

Endrôdi 1959

Parethelcus pollinarius (Forster, 1771) -

Endrödi 1969

Pelenomus canaliculatus (Fahraeus, 1843) -

Endródi 1969, Podlussány 1998

Pelenomus comari (Herbst, 1795) - Endródi

1969, Podlussány 1998

Pelenomus quadricomiger Colonnelli, 1986 -

Endródi 1969, Podlussány 1998

Pelenomus quadrituberculatus (Fabricius,

1787) - Endrődi 1969

Pelenomus waltoni (Boheman, 1843) .

Endrődi 1969, Podlussány 1998

Peritelus familiais Boheman, 1834 - Kuthy

1896, Endródi 1960, Podlussány 1998

Phloeophagus cylindrus Boheman, 1838 .

Endrôdi 1961

Phloeophagus lignarius (Marsham, 1802) -

Kuthy 1896, Endrödi I 961

Phrydiuchus speiseri (Schultze, 1897) -

Endrödi 1969 
Phrydiuchus topiarius (Germar, 1824) -

Endrödi 1969

Phyllobius arborator (Herbst, 1797) -

Endrốdi 1960

Phyllobius argentatus (Linné, 1758) -

Endródi 1960, Podlussány 1998

Phyllobius betulinus (Bechstein \&

Scharfenberg, 1805) - Endrôdi 1960,

Podlussány 1998

Phyllobius cinerascens (Fabricius, 1792) .

Endródi 1960, Podlussány 1998

Phyllobius glaucus (Scopoli, 1763) - Endrődi

1960, Podlussány 1998

Phyllobius incanus Gyllenhal, 1834 -

Endrödi 1960, Podlussány 1998

Phyllobius longipilis Boheman, 1843 -

Endrốdi 1960

Phyllobius maculicornis (Germar, 1824) -

Endrö́di 1960

Phyllobius oblongus (Linné, 1758) - Endródi 1960

Phyllobius pictus (Steven, 1829) - Endrődi

1960, Podlussány 1998

Phyllobius pomaceus Gyllenhal, 1834 -

Podlussány 1998

Phyllobius pyri (Linné, 1758) - Endrődi

1960, Podlussány 1998

Phyllobius scutellaris L. Redtenbacher, 1849 -

Endrôdi 1960, Podlussány 1998

Phyllobius seladonius Brullé, 1832 .

Endródi 1960, Podlussány 1998

Phyllobius sinuatus (Fabricius, 1801).

Endrödi 1960

Phyllobius vespertinus (Fabricius, 1792) -

Endrốdi 1960, Podlussány 1998

Phyllobius viridicollis (Fabricius, 1792) -

Endrődi 1960

Phyllobius virideaeris (Laicharting, 1781) -

Endrốdi 1960, Podlussány 1998

Phytobius leucogaster (Marsham, 1802) -

Endrốdi 1969 1961

Pissodes castaneus (De Geer, 1775) - Endrődi

Pissodes pini (Linné, 1758) - Podlussány 1998

Pissodes piniphilus (Herbst, 1797) - elsö közlés

Pissodes palidirostris (C. R. Sahlberg \& J.

Sahlberg, 1834) - Podlussány 1998

Polydrusus cervinus (Linné, 1758) - Endrődi 1959, Podlussány 1998

Polydrusus confluens Stephens, 1831 -

Endrödi 1959

Polydrusus corruscus Germar, 1824 - Endrődi

1959, Podlussány 1998

Polydrusus flavipes (De Geer, 1775) - elsö közlés 1959

Polydrusus impar Des Gozis 1882 - Endrődi
Polydrusus marginatus Stephens, 1831 Endrốdi 1959

Polydrusus mollis (Strom, 1768) - Endródi 1959

Polydrusus pallidus Gyllenhal, 1834 -

Podlussány 1998

Polydrusus picus (Fabricius, 1792) - Endrödi

1959, Podlussány 1998

Polydrusus pterygomalis Boheman, 1840 -

Endrốdi 1959, Podlussány 1998

Polydrusus sericeus (Schaller, 1783) -

Endródi 1959, Podlussány 1998

Polydrusus tereticollis (De Geer, 1775) Endrốdi 1959

Polydrusus thalassinus Gyllenhal, I834 (=merkli) - Kuthy 1896

Polydrusus tibialis Gyllenhal, 1834 - Endrődi

1959, Podlussány 1998

Polydrusus viridicinctus Gyllenhal, 1834 .

Endrốdi 1959

Poophagus sisymbrii (Fabricius, 1776) -

Endrődi 1969

Prisistus obsoletus (Germar, 1824) - Endrődi 1969

Psalidium maxillosum (Fabricius, 1792) Endrôdi 1959

Pseudocleonus cinereus (Schrank, I781) -

Endrődi 1959, Podlussány 1998

Pseudocleonus grammicus (Panzer, 1789) '.

Endrödi 1959

Pseudocoeliodes rubricus (Boheman, 1837) -

Endrốdi 1969

Pseudorchestes ermischi Dieckmann, 1958 .

Endrődi 1970

Pseudorchestes pratensis (Germar, 1821 ).

Podlussány 1998

Pseudostyphlus pillumus (Gyllenhal, 1836) Endrődi 1970

Ranunculiphilus faeculentus (Gyllenhal, 1837) - Kuthy 1896

Rhabdorhynchus varius (Herbst, 1795) -

Endrődi 1959

Rhamphus oxyacanthae (Marsham, 1802) -

Podlussány 1998

Rhamphus pulicarius (Herbst, 1795) -

Endrődi 1970, Podlussány 1998

Rhinomias austriacus Reitter, 1894 - Endrődi 1960

Rhinoncus albicinctus Gyllenhal, 1837 -

Endrödi 1969, Podlussány 1998

Rhinoncus bruchoides (Herbst, 1784) -

Endrődi 1969, Podlussány 1998

Rhinoncus castor (Fabricius, 1792) - Endrődi

1969, Podlussány 1998

Rhinoncus inconspectus (Herbst, 1795) -

Endrődi 1969, Podlussány 1998 
Rhinoncus pericarpius (Linné, 1758) .

Endrớdi 1969, Podlussány 1998

Rhinoncus perpendicularis (Reich, 1797) -

Endrödi 1969, Podlussány 1998 1959

Rhinocyllus conicus (Frölich, 1792) - Endródi

Rlynchaenus alni (Linné, 1758) - Endrödi 1970

Rhynchaenus jota (Fabricius, 1787) -

Podlussány 1998

Rhynchaenus fagi (Linné, 1758) - Endródi 1970

Rhynchaenus hortorum (Fabricius, 1792)

(=avellanae) - Endrődi 1970

Rhynchaenus hungaricus Hajóss, 1938.

Endródi 1970

Rhynchaenus pilosus (Fabricius, 1781) -

Endrödi 1970

Rhynchaenus quedenfeldtii (Gerhardt, 1865) -

Endrö́di 1970

Rhynchaenus quercus (Linné, 1758) -

Endrôdi 1970

Rhynchaenus rufus (Schrank, 1781) -

Endrödi 1970, Podlussány 1998

Rhynchaenus sparsus Fahraeus, 1843.

Endrödi 1970, Podlussány 1998

Rhynchaenus subfasciatus (Gyllenhal, 1836) -

Endrödi 1970

Rhynchaenus testaceus (O. F: Müller, 1776) -

Endrödi 1970

Rhyncolus elongatus (Gyllenhal, 1827) -

Podlussány 1998

Ruteria hypocrita (Boheman, 1837) -

Endrődi 1961, Podlussány 1998

Rutidosoma globulus (Herbst, 1795) -

Endrődi 1969

Sciaphilus asperatus (Bonsdorff, 1785) -

Endrödi 1959, Podlussány 1998 1959

Sciaphobus caesius (Hampe, 1870) - Endrôdi

Sciaphobus scitulus (Germar, I824).

Endrödi 1959

Sciaphobus squalidus (Gyllenhal, 1834) .

Endrôdi 1959

Sibinia femoralis Germar, 1824 - Endrődi 1970, Podlussány 1998

Sibinia hopffgarteni Tournier, 1873 - Endrődi 1970

Sibinia pellucens (Scopoli, 1772).

Podlussány I 998

Sibinia phalerata (Gyllenhal, 1836) -

Endrödi 1970

Sibinia primita (Herbst, 1795) - Endródi 1970

Sibinia subelliptica (Desbrochers des Loges, 1873) - Endrődi 1970

Sibinia tibialis (Gyllenhal, 1836) - Endrôdi 1970, Podlussány 1998
Sibinia unicolor (Fahraeus, 1843) - Endrődi 1970

?Simo hirticomis (Herbst, 1795) - Endrödi 1960

Simo variegatus (Boheman, 1843) - elsö közlés

Sirocalus nigrinus (Marsham, 1802) -

Endrödi 1969

Sitona ambiguus Gyllenhal, 1834 .

Podlussány 1998 1959

Sitona cambricus Stephens, 1831 - Endrödi

Sitona cylindricollis (Fahraeus, 1840) Endrődi 1959, Podlussány 1998

Sitona griseus (Fabricius, 1775) - Endrödi 1959, Podlussány 1998

Sitona hispidulus (Fabricius, 1776) -

Endrödi 1959, Podlussány 1998

Sitona humeralis Stephens, 1831 - Endródi 1959, Podlussány 1998

Sitona inops Gyllenhal, 1832 - Endródi 1959, Podlussány 1998

Sitona languidus Gyllenhal, 1834 - Endrödi 1959, Podlussány 1998

Sitona lepidus Gyllenhal, 1834 - Krécsy 1886, Endrödi 1959, Podlussány 1998

Sitona lineatus (Linné, 1758) - Endródi 1959, Podlussány 1998

Sitona longulus Gyllenhal, 1834 - Endrödi 1959

Sitona macularius (Marsham, 1802) -

Endrődi 1959, Podlussány 1998

Sitona ononidis Sharp, 1866 - Podlussány 1998

Sitona puncticollis Stephens, 1831 - Endrődi 1959, Podlussány 1998

Sitona sulcifrons (Thunberg, 1798).

Endrődi 1959, Podlussány 1998

Sitona suturalis Stephens, 1831 - Endrốdi 1959, Podlussány 1998

Sitona striatellus Gyllenhal, 1834 - Endrödi 1959, Podlussány 1998

Sitona waterhousei Walton, 1846 - Endródi 1959, Podlussány 1998 1961

Sitophilus granarius (Linné, 1758) - Endrődi 1961

Sitophilus oryzae (Linné, 1763) - Endródi

Smicronyx coecus (Reich, 1797) - elsö közlés

Smicronyx jungermanniae (Reich, 1797) -

Endrődi 1970, Podlussány 1998

Smicronyx reichii (Gyllenhal, 1836) -

Endródi 1970

Smicronyx striatipennis Tournier, 1874 - elsố közlés

Sphenophorus abbreviatus (Fabricius, 1787) Endrődi 1961 1961 
Sphenophorus striatopunctatus (Goeze, 1777) Endrödi I961

Stenocarus cardui (Herbst, 1784) - Endródi 1969

Stenocarus ruficornis (Stephens, 1831) -

Endrôdi 1969

Stereocorynes truncorum (Germar, 1824) -

Kuthy 1896

Stereonychus fraxini (De Geer, 1775) -

Endródi 1970, Podlussány 1998

Stomodes gyrosicollis Boheman, 1843 .

Endrôdi 1960

Strophosoma capitatum (De Geer, 1775) -

Endrödi 1959

Strophosoma faber (Herbst, 1785) - Kuthy

1896, Endródi 1959

Strophosoma melanogrammum (Forster, 1771) -

Endrōdi 1959, Podlussány 1998

Tachyerges decoratus (Germar, 1821) -

Endrôdi 1970, Podlussány 1998

Tachyerges pseudostigma Tempére, 1982 -

Podlussány 1998

Tachyerges salicis (Linné, 1758) - Endrödi 1970, Podlussány 1998

Tachyerges stignta (Cicrmar, 182 I) - Endrödi

1970, Podlussány 1998

Tanymecus dilaticollis Gyllenhal, 1834 -

Endrö́di 1959, Podlussány 1998

Tanymecus palliatus (Fabricius, 1787) -

Endrōdi 1959, Podlussány 1998

Tanysphyrus lemnae (Paykull, 1792) -

Endrődi 1961, Podlussány 1998

Tapinotus sellatus (Fabricius, 1794) -

Endrôdi 1969, Podlussány 1998

Thamiocolus pubicollis (Gyllenhal, 1837) -

Endrödi 1969

?Thamiocolus sahlbergi (Sahlberg, 1845) -

Endrôdi 1969

Thamiocolus signatus (Gyllenhal, 1837) -

Endródi 1969

Thamiocolus viduatus (Gyllenhal, 1837) -

Endrödi 1969

Thryogenes atrirostris Lohse, 1992.

Podlussány 1998 1970

Thryogenes festucae (Herbst, 1795) - Endrődi

Thryogenes nereis (Paykull, 1800) Podlussány 1998

Thryogenes scirrhosus (Gyllenhal, 1836) Endródi 1970

Trachodes hispidus (Linné, 1758) -

Podlussány 1998

Trachyphloeus alternans Gyllenhal, 1834 -

Endrödi 1959

Trachyphloeus angustisetulus Hansen, 1915 - elsố közlés

Trachyphloeus aristatus (Gyllenhal, 1827) -

Endródi 1959, Podlussány 1998

Trachyphloeus asperatus Boheman, 1843 -

Endródi 1959

Trachyphloeus bifoveolatus (Beck, 1817) -

Endródi 1959

Trachyphloeus inermis Boheman, I843.

Endrốdi 1959

Trachyphloeus parallelus Seidlitz, 1868 .

Endrödi 1959

Trachyphloeus spinimanus Germar, 1824 .

Endrödi 1959

Trachyphloeus ventricosus Germar, 1824 -

Endrödi 1959

Trichosirocalus horridus (Panzer, 1801) -

Endrödi 1969

Trichosirocalus rufulus (Dufour, 1851) Endrödi 1969

Trichosirocalus troglodytes (Fabricius, 1787) -

Endródi 1969

Tropiphorus micans Boheman, 1842 .

Endrödi 1959

Tychius aureolus Kiesenwetter, 1851 - Kuthy

1896 , Endrödi 1970

Tychius breviusculus Desbrochers des Loges,

1873 - Endrôdi 1970, Podlussány 1998

Tychius crassirostris Kirsch, 1871 - Endródi 1970

Tychius cuprifer (Panzer, 1799) - Endrődi 1970, Podlussány I 998

Tychius flavus Becker, 1864 - Endrödi 1970

Tychius junceus (Reich, 1797) - Endródi 1970 1970

Tychius lineatulus Stephens, 1831 - Endrődi

Tychius medicaginis Ch. Brisout de Barneville, 1862 - Endródi I970, Podlussány 1998

Tychius meliloti Stephens, 1831 - Endrôdi 1970, Podlussány 1998 1970

Tychius parallelus (Panzer, 1794) - Endrödi

Tychius picirostris (Fabricius, 1787) -

Endrōdi 1970, Podlussány 1998

Tychius polylineatus (Germar, 1824) Endrődi 1970

Tychius pumilus Ch. Brisout de Barneville, 1862 - Endródi 1970, Podlussány 1998

Tychius quinquepunctatus (Linné, 1758) Endrődi 1970, Podlussány 1998

Tychius schneideri (Herbst, 1795) - Endródi 1970

Tychius squamulatus Gyllenhal, 1836 -

Kuthy 1896, Endrődi 1970

Tychius stephensi Schönherr, 1836 - Endrödi 1970, Podlussány 1998

Tychius subsulcatus Tournier, 1873 - Endrödi 1970 
Tychius tibialis Boheman, 1843 - Endrödi 1970

Tychius trivalis Boheman, 1843 - Endródi 1970

Zacladus asperatus (Gyllenhal, 1837) -

Endrödi 1969

Zacladus geranii (Paykull, 1800) - Endrődi 1969, Podlussány 1998

\section{Apionidae}

Acanephodus onopordi (Kirby, 1808) -

Podlussány 1998

Apion cruentatum Walton, 1844 .

Podlussány 1998

Apion frumentarium (Linné, 1758) -

Podlussány 1998 1998

Apion haematodes Kirby, 1808 - Podlussány

Apion rubiginosum Grill, 1893 - Podlussány 1998

Aspidapion radiolus (Marsham, 1802) -

Krécsy 1886, Podlussány 1998

Aspidapion aeneum (Fabricius, 1775) -

Kuthy 1896, Podlussány 1998

1886

Aspidapion validum (Germar, 1817) - Krécsy

Catapion jaffense (Desbrochers des Loges,

1895) - elsö közlés

Catapion pubescens (Kirby, 1811) - elsö közlés

Catapion seniculus (Kirby, 1808) .

Podlussány 1998

Ceratapion basicorne (Illiger, 1807) - elsố közlés

Ceratapion gibbirostre (Gyllenhal, 1813) -

Podlussány 1998

Ceratapion penetrans (Germar, 1817) .

Kuthy 1896, Podlussány 1998

Cyanapion afer (Gyllenhal, 1833).

Podlussány 1998

Cyanapion columbinum (Germar, 1817) -

Podlussány 1998

Cyanapion gyllenhalii (Kirby, 1808) -

Podlussány 1998

Cyanapion platalea (Germar, 1817) - elsố közlés

Dieckmanniellus gracilis (L. Redtenbacher,

1849) - Endródi 1970

Dieckmanniellus helveticus (Tournier, 1867) -

Endrốdi 1970

Dieckmanniellus nitidulus (Gyllenhal, 1838) -

Endródi 1970

Diplapion confluens (Kirby, 1808) - elsó közlés

Diplapion detritum (Mulsant \& Rey, 1859) -

Podlussány 1998

Diplapion stolidum (Germar, 1817) - elsö közlés

Eutrichapion ervi (Kirby, 1808) - elsö közlés közlés

Eutrichapion facetum (Gyllenhal, 1839) - elsó

Eutrichapion gribodoi (Desbrochers des
Loges, 1896) - elsö közlés

Eutrichapion melancholicum (Wencker, 1864) -

Podlussány 1998

Eutrichapion viciae (Paykull, 1800) -

Podlussány 1998

Eutrichapion vorax (Herbst, 1797) - elsó közlés

Eutrichapion punctigerum (Paykull, 1792) -

Krécsy 1886, Podlussány 1998

Exapion comiculatum (Germar, 1817) - elsö közlés

Exapion difficile (Herbst, 1797) - elsố közlés

Exapion elongatulum (Desbrochers des Loges, 1891) - elsố közlés

Exapion formaneki (Wagner, 1929) - elsố közlés

Exapion fuscirostre (Fabricius, 1775) -

Podlussány 1998

Hemitrichapion pavidum (Germar, 1817) -

Podlussány 1998

Hemitrichapion reflexum (Gyllenhal, 1833) -

elsố közlés

Holotrichapion aestimatum (Faust, 1891) -

Podlussány 1998

Holotrichapion pisi (Fabricius, 1801) -

Krécsy 1886, Podlussány 1998

Ischnopterapion fallens (Marseul, 1888) $-\mathrm{el}$ sö közlés

Ischnopterapion loti (Kirby, 1808) -

Podlussány 1998

Ischnopterapion virens (Herbst, 1797) -

Krécsy 1886, Podlussány 1998

Kalcapion pallipes (Kirby, 1808) - elsố közlés

Malvapion malvae (Fabricius, 1775) -

Podlussány 1998

Melanapion minimum (Herbst, 1797) -

Podlussány 1998

Mesotrichapion punctirostre (Gyllenhal, 1839) - első közlés

Microon sahlbergi (C. R. Sahlberg, 1835) -

Endrödi 1970

Nanomimus hemisphaericus (Olivier, 1807) -

Endrödi 1970

Nanophyes brevis Boheman, 1845 - Endrödi

1970, Podlussány 1998

Nanophyes globiformis Kiesenwetter, I 864 -

Endrödi 1970

Nanophyes globulus (Germar, 1821) -

Endrődi 1970

Nanophyes marmoratus (Goeze, 1777) -

Endrődi 1970, Podlussány 1998

Omphalapion dispar (Germar, 1817) - elsö közlés közlés

Omphalapion hookerorum (Kirby, 1808) - elsó

Omphalapion laevigatum (Paykull, 1792) -

Krécsy 1886

Oxystoma craccae (Linné, 1767) - Podlussány 1998 
Oxystoma dimidiatum (Desbrochers des Loges, 1897) - Podlussány 1998

Oxystoma ochropus (Germar, 1818) - elsö közlés

Oxystoma pomonae (Fabricius, 1798) - elsó közlés

Perapion affine (Kirby, 1808) - Podlussány 1998

Perapion curtirostre (Germar, 1817) - elsó közlés

Perapion violaceum (Kirby, 1808) -

Podlussány 1998 közlés

Phrissotrichum rugicolle (Germar, 1817) - elsö

Protapion apricans (Herbst, 1797) - Krécsy 1886, Podlussány 1998

Protapion assimile (Kirby, 1808) -

Podlussány 1998

Protapion dissimile (Germar, 1817) -

Podlussány 1998

Protapion filirostre (Kirby, 1808) - Krécsy 1886, Podlussány 1998

Protapion fulvipes (Fourcroy, 1785) -

Podlussány 1998

Protapion nigritarse (Kirby, 1808) - Krécsy 1886, Podlussány 1998 közlés

Protapion ononidis (Gyllenhal, 1827) - elsố

Protapion ruficrus (Germar, 1817) Podlussány 1998

Protapion trifolii (Linné, 1768) - Podlussány 1998

Protapion varipes (Germar, 1817) Podlussány 1998 sö közlés

Protopirapion atratulum (Germar, 1817) - el-

Pseudapion fulvirostre (Gyllenhal, 1833) - elsố közlés

Pseudapion rufirostre (Fabricius, 1775) Podlussány 1998

Pseudoperapion brevirostre (Herbst, 1797) Podlussány 1998

Pseudoprotapion astragali (Paykull, 1800) Podlussány 1998

Pseudoprotapion elegantulum (Germar, 1818) elsö közlés

Pseudostenapion simum (Germar, 1817) Podlussány 1998

Rhopalapion longirostre (Olivier, 1807) Krécsy 1886, Podlussány 1998 közlés

Squamapion atomarium (Kirby, 1808) - elsó

Squamapion cineraceum (Wencker, 1864) Podlussány 1998

Squamapion elongatum (Germar, 1817) - elsố közlés

Squamapion flarimanum (Gyllenhal, 1833) Podlussány 1998 közlés

Squamapion hoffmanni (Wagner, 1930) - elsó

Squamapion oblivium (Schilsky, 1902) - elsó közlés

Squamapion picinum (Kirby, 1808) -

Podlussány 1998

Stenopterapion intermedium (Eppelsheim, 1875) - elsö közlés

Stenopterapion meliloti (Kirby, 1808) - Kuthy 1896, Podlussány 1998

Stenopterapion tenue (Kirby, 1808) -

Podlussány 1998

Synapion ebeninum (Kirby, 1808) -

Podlussány 1998

Taeniapion rufulum (Wencker, 1864) - elsó közlés

Taeniapion urticarium (Herbst, 1784) Podlussány 1998

Trichapion simile (Kirby, 1811) - Podlussány 1998

Trichopterapion holosericeum (Gyllenhal, 1833) - Krécsy 1886, Podlussány 1998

\section{Anthribidae}

Anthribus albinus (Linné, 1758) -

Podlussány 1998

Brachytarsus nebulosus (Forster, 1771) -

Endrődi 1963, Podlussány 1998

Dissoleucas niveirostris (Fabricius, 1798) -

Endrődi 1963, Podlussány 1998

Enedreytes sepicola (Fabricius, 1792) -

Endródi 1963, Podlussány 1998

Phaeochrotes cinctus (Paykull, 1800) -

Endrődi 1963

Platyrhinus resinosus (Scopoli, 1763) -

Endrôdi 1963, Podlussány 1998

?Rhaphitropis oxyacanthae (Ch. Brisout de

Barneville, 1863) - Endrődi 1963

Rhaphitropis marchicus (Herbst, 1797) -

Endrôdi 1963, Podlussány 1998

Tropideres albirostris (Herbst, 1784) -

Endrődi 1963

Ulorhinus bilineatus (Germar, 1818) -

Endrődi 1963, Podlussány 1998

\section{Attelabidae}

Apoderus coryli (Linné, 1758) - Endrődi 1957, Podlussány 1998

Apoderus erythropterus (Gmelin, 1790) -

Endródi 1957, Podlussány 1998

Attelabus nitens (Scopoli, 1763) - Endrôdi 1957

Byctiscus betulae (Linné, 1758) - Endrődi

1957, Podlussány 1998

Byctiscus populi (Linné, 1758) - Endrödi 1957

Caenorhinus aeneovirens (Marsham, 1802) -

Endródi 1957, Podlussány 1998 
Caenorhinus aequatus (Linné, 1767).

Endrôdi 1957, Podlussány 1998

Caenorhinus germanicus (Herbst, 1797) -

Endrôdi 1957, Podlussány 1998

Caenorhinus interpunctatus (Stephens, 1831)-

Endrôdi 1957

Caenorhinus pauxillus (Germar, 1824) -

Endrődi 1957, Podlussány 1998

Cimberis attelaboides (Fabricius, 1787) -

Endrödi 1957

Deporaus betulae (Linné, 1758) - Endrödi

1957, Podlussány 1998 1957

Deporaus tristis (Fabricius, 1794) - Endrődi

Doydirhynchus austriacus (Olivier, 1807) -

Endródi 1957, Podlussány 1998

Inrolvulus aethiops Bach, 1854 - Endrődi 1957

Involvulus caeruleus (De Geer, 1775) -

Endrödi 1957

Lasiorhynchites cavifrons (Gyllenhal, 1833) -

Endrödi 1957, Podlussány 1998

Lasiorhynchites olivaceus (Gyllenhal, 1833) -

Endrödi 1957

Lasiorhynchites sericeus (Herbst, 1797) -

Endrốdi 1957

Nemonyx lepturoides (Fabricius, 1801) -

Kuthy 1896, Endrödi 1957

Pselaphorhynchites nanus (Paykull, 1792) -

Endrödi 1957

Pselaphorhynchites tomentosus (Gyllenhal, 1839) - Endrődi 1957, Podlussány 1998

Rhynchites auratus (Scopoli, 1763).

Endrödi 1957

Rhynchites bacchus (Linné, 1758) - Endrödi

1957, Podlussány 1998

Scolytidae

Crypturgus cinereus (Herbst, 1793).

Endrödi 1958

Crypturgus pusillus (Gyllenhal, 1813) -

Endrödi 1958, Podlussány 1998

Dryocoetes autographus (Ratzeburg, 1837) -

Endrốdi 1958

Dryocoetes hectographus Reitter, 1913.

Endrödi 1958

Dryocoetes pillosus (Fabricius, 1792) -

Endrödi 1958

Ernoporicus caucasicus (Lindemann, 1876) -

Endrödi 1958 1958

Ernoporicus fagi Fabricius, 1798) - Endrödi 1958

Enoporus tiliae (Panzer, 1793) - Endrődi

Hylastes angustatus (Herbst, 1793) -

Endrődi 1958, Podlussány 1998
Hylastes ater (Paykull, 1800) - Endródi 1958

Hylastes cunicularius Erichson, I 836.

Endrôdi 1958

Hylastes linearis Erichson, 1836 - Endrödi 1958

Hylastinus obscurus (Marsham, 1802). Endrôdi 1958

Hylastes opacus Erichson, 1836 - Endrődi 1958, Podlussány 1998

Hylurgops palliatus (Gyllenhal 1813) -

Endrődi 1958, Podlussány 1998

Hylurgus ligniperda (Fabricius, 1792) -

Endródi 1958, Podlussány 1998 1958

Ips acuminatus (Gyllenhal, 1827) - Endródi.

Ips sexdentatus (Börner, I767) - Krécsy

1886, Endródi 1958, Podlussány 1998

Ips typographus (Linné, 1758) - Endrödi 1958 Leperisinus varius (Fabricius, 1775) -

Endrödi 1958, Podlussány 1998

Lymantor coryli (Perris, 1853) - Endrödi 1958

Orthotomicus laricis (Fabricius, 1792).

Endrôdi 1958, Podlussány [998

Orthotomicus proximus (Eichhoff, 1868) -

Endrödi 1958, Podlussány 1998

Orthotomicus robustus (Knotek, 1899) .

Podiussány 1998

Orthotomicus suturalis (Gyllenhal, 1827) -

Endrődi 1958, Podlussány 1998

?Phloeophthorus brevicollis Kolenati, 1846 -

Endródi 1958

Phloesinus aubei (Perris, 1855) - Endrödi 1958

Phloesinus thujae (Perris, 1855) - Endrödi 1958

Pityogenes quadridens (Hartig, 1834) -

Endródi 1958

Pityokteines curvidens (Germar, 1824) -

Endródi 1958

Pityokteines porontzowi (Jacobson, 1895) -

Endrốdi 1958

Pityophthorus pubescens (Marsham, 1802) -

Endródi 1958

Pteleobius kraatzi (Eichoff, 1864) - Kuthy

1896, Endrōdi 1958

Pteleobius vittatus (Fabricius, 1787) -

Endródi 1958, Podlussány 1998

Scolytus intricatus (Ratzeburg, 1837) -

Endrödi 1958

Scolytus mali (Bechstein, 1805) - Endrödi 1958

Scolytus multistriatus (Marsham, 1802) -

Endrödi 1958, Podlussány 1998

Scolytus pygmaeus (Fabricius, 1787) .

Endrödi 1958

Scolytus ratzeburgii Janson, 1856 - Kuthy 1896, Endrődi 1958 
Scolytus rugulosus (Ph. W. J. Müller, 1818) Endrödi 1958

Scolytus scolytus (Fabricius, 1775) - Kuthy 1896, Endrödi 1958

Taphrorychus bicolor (Herbst, 1793) Endrődi 1958

Taphrorychus hirtellus Eichhoff, 1879 Endrö́di 1958

Tomicus piniperda (Linné, 1758) - Endrödi 1958, Podlussány 1998

Trypodendron domesticum (Linné, 1758) . Endrớdi 1958

Trypodendron lineatum (Olivier, 1795) Endrö́di 1958

Trypodendron signatum (Fabricius, 1792) Endrődi 1958

Trypophloeus granulatus (Ratzeburg, 1837) Endrö́di 1958
Trypophloeus rybinskii (Reitter, 1895) Podlussány 1998

Xyleborinus saxesenii (Ratzeburg, 1837) Endrő́di 1958

Xyleborus cryptographus (Ratzeburg, 1837) Endrö́di 1958

Xyleborus dispar (Fabricius, 1792) - Endrődi 1958, Podlussány 1998

Xyleborus monographus (Fabricius, 1792) Endrớdi 1958

Xyleborus pfeilii (Ratzeburg, 1837) . Podlussány 1998

Xylocleptes bispinus (Duftschmid, 1825) Endrödi 1958, Podlussány 1998

Platypodidae

Platypus cylindrus (Fabricius, 1792) Endródi 1958

\section{1. táblázat: A Somogyból ismert Curculionoidea fajok családonkénti megoszlása}

\begin{tabular}{|l|c|}
\hline Család & Fajszám \\
\hline Curculionidae & 570 \\
\hline Apoinidae & 97 \\
\hline Anthribidae & 10 \\
\hline Attelabidae & 24 \\
\hline Scolytidae & 55 \\
\hline Platypodidae & 1 \\
\hline összesen: & 757 \\
\hline
\end{tabular}




\title{
Irodalom
}

EN1DRŐd, S. 1957: Az eszelény-félék (Attelabidae) kárpátmedencei lelóhelyadatai. - Folia ent. hung. I0: $48 \mathrm{I}-494$.

ENDRŐ́n, S. 1958b: A szubogarak (Scolytidae) kárpátmedencei lelóhelyadatai. - Folia Entomologica Hungarica $11: 21-43$.

ENDRÖıI, S. 1959a: Az ormányosbogarak (Curculionidac) kárpátmedencei lelóhelyadatai, I. Folia ent. hung. 12: 215-262.

ENIDRÖID, S. 1960b: $\Lambda \%$ ormányosbogarak (Curculionidac) kárpátmedencei lclóhelyadatai, II. Folia lc): Az ormányosbogarak (Curculionidae) kárpátmedencei lelóhelyadatai, III. - Folia ent. hung. 14: 279-3 I 6 .

ENDRÓDI, S. 1963a: ^z orrosbograrak (Anthribidae) kárpátmedencei lelöhelyadatai. - Folia ent. hung. I 6: 137-144.

ENIIRŐDI, S. 1969: A\% ormányosbogarak (Curculionidae) kárpátmedencei lelóhelyadatai, IV. Folia ent. hung. 22: $311-348$

ENDRÖDI, S. 1970: Az ormányosbogarak (Curculionidae) kárpátmedencei lelöhelyadatai, V. - Folia ent. hung. 23: 349-400.

KRÉCSY, B. 1886: Adatok Somogy megye bogárfaunájához. - Rovartani Lapok 3: 146-148.

Kurtr, D. [1896 - 1897]: Coleoptera. - In: A Magyar Birodalom Állatvilága (Fauna Regni Hungariae). A K. M. Természettudományi Társulat, Budapest, 140-174.

Podlussíny, A. 1996: Magyarország ormányosalkatú bogarainak fajlistája (Coleoptera: Curculionoidea) - Folia ent. hung. 57: 197-225.

Ponlussány, A. 1998: A Duna-Dráva Nemzeti Park Dráva-mente Curculionoidea faunájának alapvetése. - Folia ent. hung. 59: 271-315.

\section{Der Katalog der Cuculionoidea Arten des Komitates Somogy (Coleoptera: Curculionoidea)}

\author{
AtTrilla PodlussánY
}

Der Autor hat die Angaben der im Komitat Somogy ab 1886 bis heute gesammelten Curculionoidea Arten zusammengebracht. Unsere Ahnen haben keine das ganze Territorium des Komitats Somogy umfassende Forschung angestellt. Dementsprechend ist die Beschreibung von 757 Arten ein bedeutender Erfolg, was den Reichtum der Flora dieses Komitats beweist, da all die Rüsselkäfer Pflanzenfresser sind. Der Verfasser kündigt eine für die ungarische Fauna neue Art an - Ceutorhynchus fulvitarsis Gougelet \& H. Brisout de Barneville, 1860 - welche bisher nur in dem Komitat Somogy gefunden worden ist.

Anschrift des Verfassers:

Attila PODLUSSÁNY

H-l Ill Budapest

Bercsényi u. 10.

UNGARN 